\title{
Non-resonant RF and microwave response: A novel technique for the characterization of superconducting materials
}

\author{
S V BHAT \\ Department of Physics, Indian Institute of Science, Bangalore 560012, India
}

\begin{abstract}
When examined using continuous wave electron paramagnetic resonance and nuclear magnetic resonance spectrometers, the high $T_{\mathrm{c}}$ superconductors give rise to intense, low field, 'non-resonant' absorption signals in the superconducting state. This phenomenon can be used as a highly sensitive, contactless technique for the detection and characterization of superconductivity even in samples containing only minute amounts of the superconducting phase. Further, it can also be applied to the determination of material parameters of interest such as $J_{\mathrm{c}}$ and $H_{\mathrm{c} 2}$ in addition to being a powerful way of distinguishing between weak-link superconductivity and bulk superconductivity. The details of these aspects are discussed.
\end{abstract}

Keywords. Non-resonant microwave absorption; high-temperature superconductors; weak-links; fluxon motion.

\section{Introduction}

The study of electrodynamic properties of superconductors subjected to magnetic fields is important from both the fundamental and technical points of view. Not only can it provide a test for any plausible microscopic theory of superconductivity but it can also yield information on the material parameters important for many of the technological applications. After the advent of the high- $T_{c}$ superconductors (Bednorz and Muller 1986; Chu et al 1987), a new, highly sensitive method of studying the response of superconductors to electromagnetic radiation has come into vogue. It is based on the observation (Bhat et al 1987a, b) that when examined using conventional continuous wave (CW) electron paramagnetic resonance (EPR) and nuclear magnetic resonance (NMR) spectrometers, the new superconductors exhibit at low magnetic fields, in the superconducting state, intense signals even with minuscule amounts of the samples. The high sensitivity of the technique arises because of the magnetic field modulation and lock-in detection commonly used in the CW EPR and NMR spectrometers. In the absence of hysteresis, the technique yields the field derivative, $\mathrm{d} P / \mathrm{d} H$, of the power absorbed by the sample. The properties of these derivative signals are very sensitively dependent on temperature, applied magnetic field, and quite often on the nature and quality of the samples. First observed in ceramic samples of $\mathrm{YBa}_{2} \mathrm{Cu}_{3} \mathrm{O}_{7-x}$ and $\mathrm{Y}_{0.75} \mathrm{Lu}_{0.25} \mathrm{Ba}_{2} \mathrm{Cu}_{3} \mathrm{O}_{7-x}$ by us (Bhat et al 1987a, b) this phenomenon is found to occur in different forms of these materials like single crystals and thin films as well as the ceramics. Keeping up with the pace of the activity in other areas of superconductivity research, over the last seven years a large number $(>150)$ of papers have appeared on this subject (for an early review see Blazey 1990). As a consequence, many of the earlier uncertainties regarding the nature of the phenomenon have been clarified. In this article, we plan to indicate the applications of the technique to two specific cases of importance to materials research, namely the measurement of the critical current density $J_{c}$ and the characterization of the samples for the 
presence of weak links and the determination of the nature of the weak links that are present.

\section{Early experimental results}

The first observation of the non-resonant microwave absorption phenomenon in the high- $T_{\mathrm{c}}$ superconductors was actually made during an attempt to study the EPR of $\mathrm{Cu}^{2+}$ in ceramic samples of $\mathrm{YBa}_{2} \mathrm{Cu}_{3} \mathrm{O}_{7-x}$. The attempt was motivated by the fact that EPR is known, from the earlier studies of conventional superconductors (Baberschke 1976), to be one of the useful techniques to study superconductivity. In our initial experiments (Bhat et al 1987a) it was observed that a signal with an anisotropic $g$ parameter of $\sim 2$ and attributable to $\mathrm{Cu}^{2+}$ ion was present in the normal state of the compound. The signal disappeared below $T_{c}$ accompanied by an increase in the spectrometer noise. [Now it is well established that a pure single-phase superconducting material does not give rise to any $\mathrm{Cu}^{2+} \mathrm{EPR}$ signal either in the normal state or in the superconducting state. Some of the possible reasons for this 'EPR silence' in these materials are discussed by Mehran and Anderson (1989). Very interestingly, however, an intense signal (figure 1) appeared just below $T_{\mathrm{c}}$ centred around zero magnetic field and continued to evolve in the superconducting state. The following properties of this signal were noted:

(i) The signal was very narrow $(\sim 10 \mathrm{Oe})$ and much stronger than the $g \sim 2$ signal and disappeared on warming the sample to $T>T_{\mathrm{c}}$.

(ii) During warming, while the intensity of the signal went on decreasing, a few degrees below $T_{\mathrm{c}}$ the phase of the signal reversed.

(iii) For low modulation amplitudes, the signals for the forward and the backward magnetic field sweeps showed pronounced hysteresis, in as much as the phases of signals for the two opposite scans were completely opposite.

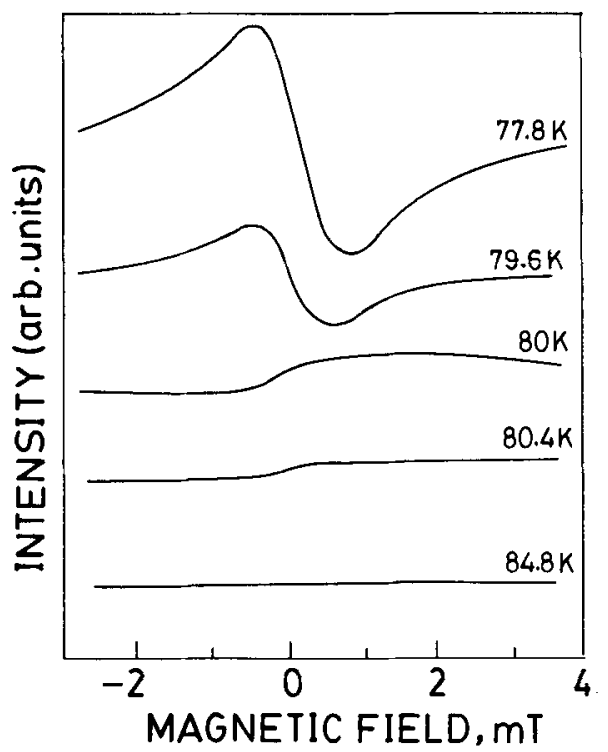

Figure 1. Near-zero-field signal of $\mathrm{YBa}_{2} \mathrm{Cu}_{3} \mathrm{O}_{7-x}$ as a function of temperature in the superconducting state. 
Immediately following this preliminary report, in a more detailed publication (Bhat et al 1987b) the phenomenon was characterized further not only in the microwave range but also in the rf range between 8 and $22 \mathrm{MHz}$. The fact that the signal occurred centred at zero field for frequencies different by three orders of magnitude indicated that one was essentially dealing with a non-resonant phenomenon. The strong hysteretic behaviour dependent on the modulation amplitude observed earlier was confirmed. As a function of decreasing temperature, the intensity of the signal was observed to go on increasing reaching a plateau around $77 \mathrm{~K}$. The temperature dependence was tracked in more detail at of frequencies and a broadening of the signal as a function of decreasing temperature was observed (figure 2). The signal width was also found to depend upon the particle size. With fine powder samples, a large number of narrow signals with peak-to-peak widths of a fraction of an Oe were observed. In the rf range, the frequency change of the oscillator on going from the normal to the superconducting state was measured and a large increase in the frequency $(\sim 4 \%)$ was observed. This change was attributed to the diamagnetic response of the sample and the absorption signals were concluded to occur consequent to dissipation in the Josephson junctions. Further, a sensitivity of the signal intensity to the presence of oxygen was found. It was observed that the signal intensity was much smaller for an evacuated sample. When exposed to air or oxygen, the intensity built up again. A part of this increase in the intensity could be attributed to better and faster equilibration of temperature. The oxygen or air leads to more effective thermal contact between the grains of the powder sample and also between the sample and the walls of the container kept at $77 \mathrm{~K}$. However, in an experiment carried out (Bhat et al, unpublished) to check this aspect where the evacuated sample was kept at liquid nitrogen temperatures for a long time $(\sim 1 / 2 \mathrm{~h})$ and then air was let

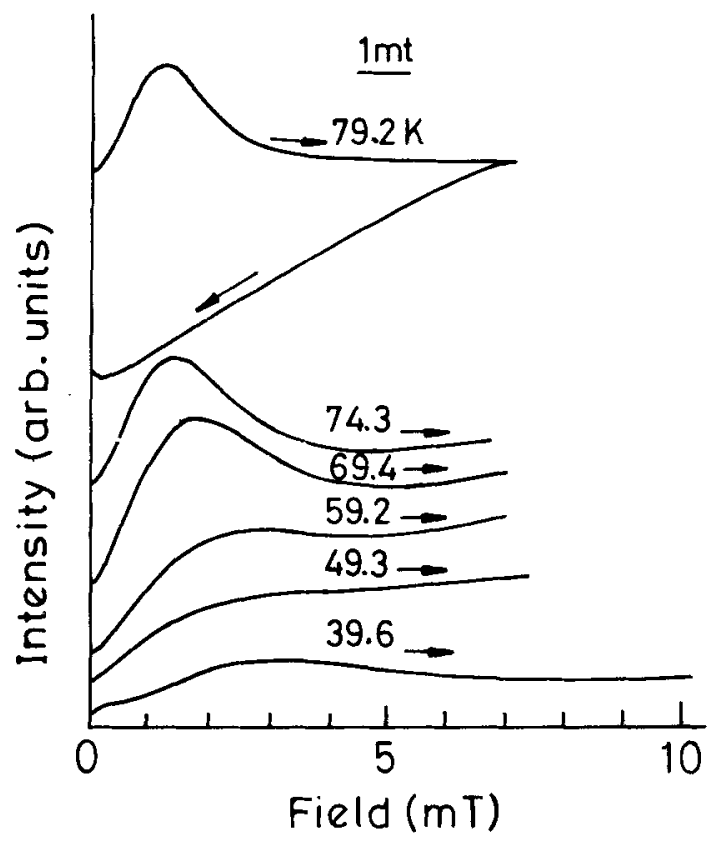

Figure 2. Derivative signals recorded at $13.35 \mathrm{MHz}$ at different temperatures with a $\mathrm{CW}$ NMR spectrometer. 
in, an increase in the signal intensity was found. It must also be noted that Blazey et al (1988), in a subsequent study of machined, then lightly etched and finally anodically oxidized $\mathrm{Nb}$ metal, found that the signal disappeared on etching and returned after oxidation.

Shortly after our first report and almost contemporaneously with our second, a large number of studies in the microwave range using the similar technique of the use of an ESR spectrometer were reported and essentially confirmed our major observations (Blazey et al 1987; Dulcic et al 1987; Durney et al 1987; Khachaturyan et al 1987; Rettori et al 1987; Stankowski et al 1987; Peric et al 1988). In the following pages, we shall discuss some of these various aspects in greater detail after a brief discussion of the experimental techniques used with a view to providing a perspective for understanding the nature of these non-resonant signals recorded with the magnetic resonance spectrometers.

\section{Experimental techniques}

The techniques of EPR (Pilbrow 1990) and NMR (Abragam 1961) are very well established local probe methods which have a history of more than four decades. They have found a wide variety of applications in various fields of science and engineering. There are therefore a large number of textbooks, monographs and treatises available treating the subjects at various levels. A special mention must be made of the book by Poole (1983) on the experimental techniques in ESR which is a very detailed exposition on the subject. In contrast, the practice of CW NMR is almost completely extinct because of the versatility of the pulsed Fourier transform NMR spectrometers. In this context, we may point out that our home-made $\mathrm{CW}$ NMR spectrometer enabled us to provide unique experimental results (to be described later) by way of comparison of microwave and if responses. Excellent description of the experimental aspects of the CW NMR spectrometers is available in a number of earlier books in the field (Andrew 1955).

Even though ESR and NMR differ in the actual techniques because of the different instrumentational requirements of the generation and detection of the two frequency ranges differing in three orders of magnitude, they are, however, based on essentially the same phenomenon of observations of the resonant absorption of electromagnetic radiation of appropriate frequency caused by transitions between the Zeeman split energy levels. In the CW EPR and NMR spectrometers the samples placed in either the resonant cavity or the resonant coil of the tank circuit are usually subjected to three different magnetic fields such that the total field $H$ applied to the sample is given by

$$
H=H_{0} \mathrm{z}+H_{1} \cos \omega_{1} t \mathrm{x}+H_{\mathrm{m}} \cos \omega_{\mathrm{m}} t \mathrm{z},
$$

where the static field $H_{0} \sim 0.5 \mathrm{~T}$ to a few $\mathrm{T}$ for $\mathrm{NMR}, 0.3 \mathrm{~T}$ for EPR at X-band, $1.2 \mathrm{~T}$ for EPR at Q-band; the resonant frequency $2 \pi \omega_{1}=\mathrm{a}$ few $\mathrm{MHz}$ to a few hundred MHz for NMR, 9.5 GHz for X-band EPR, $35 \mathrm{GHz}$ for Q-band EPR; the modulation frequency $\omega_{\mathrm{m}}=\mathrm{a}$ few tens of $\mathrm{Hz}$ for NMR and usually $100 \mathrm{kHz}$ for EPR. The strengths of $H_{1}$ and $H_{\mathrm{m}}$ fields may vary from a fraction of an Oe to a few Oe. As a consequence of the magnetic field modulation and the phase-sensitive detection schemes normally employed in these spectrometers, the signal recorded is in the derivative form. In the 
case of an EPR experiment, the response of the sample to these fields is detected as a change in the reflected microwave power level resulting from the absorption of the microwaves by the sample on crossing through the resonance consequent to scanning the dc magnetic field. The reflected microwaves are incident on a detector diode which normally operates in the 'linear law' regime and outputs a voltage proportional to the square root of the absorbed microwave power. The CW NMR spectrometers operating in the single-coil configuration normally yield signals which are changes in the rf levels of the tank circuit which can be looked upon as a consequence of a change in the shunt resistance of the circuit on resonance. In both the cases, the signals can be expressed in terms of the quality factor $Q$ of the resonator: i.e. the signal voltage $\Delta V$ is given by

$$
\Delta V=-V_{0} \zeta\left(4 \pi \chi^{\prime \prime}+i 4 \pi \chi^{\prime}\right) Q
$$

where $V_{0}$ is the if level in the absence of resonance, $\zeta$ the filling factor, and $\chi^{\prime}$ the real part of the complex susceptibility of the sample. Since $V_{0}$ is real, to first order, only $\chi^{\prime \prime}$ is detected. The imaginary component $-\mathrm{i} V_{0} Q 4 \pi \chi^{\prime}$ is said to be in quadrature and affects the magnitude of the loss only to the second order in $\chi^{\prime}$. However it can lead to a change in the frequency of the resonator which also can be used to detect (Bhat et al 1987b) and characterize the superconducting state.

\section{Critical current density measurements of $\mathrm{YBa}_{2} \mathrm{Cu}_{3} \mathrm{O}_{7-x}$ thin films by non-resonant rf absorption method}

As mentioned earlier, it is normal practice in the CW EPR and NMR spectrometers to use low-frequency magnetic field modulation and phase-sensitive detection which result in the field derivative of the absorption being recorded. Here we show that this fact can also be used to determine the critical current density $J_{c}$ of superconducting materials (Srinivasu et al 1991). We study thin-film samples of the $\mathrm{YBa}_{2} \mathrm{Cu}_{3} \mathrm{O}_{7-x}$ (123) compound and measure the intensity dependence of the signals on the amplitude of the modulating field and interpret it in terms of the Bean critical state model (Bean $1962,1964)$. The effective $J_{c}$ values thus obtained, when scaled to take into account the recent observations (Baczewski et al 1991) that the critical current is sustained primarily by the normal gradient in the in-plane component of the flux density, yield true $J_{c} s$ that compare satisfactorily with independently measured transport $J_{c}$ values.

The 123 films were fabricated by pulsed laser deposition method. The films were characterized by X-ray diffraction. The resistance was measured by standard fourprobe method. Transport $J_{c}$ measurements were carried out on 80-micron ribbons of the films. The ribbons were made by laser dry-etching of the films using tungsten wire masks.

A home-assembled CW NMR spectrometer based on a Robinson-type level-limited oscillator working at a nominal frequency of $10 \mathrm{MHz}$ was used to record the nonresonant if response (Bhat et al 1987b). Field modulation at $87 \mathrm{~Hz}$ was used and the lock-in detection yielded the derivatives of the rf loss signals. The static field $H_{0}$ was swept in the region \pm 100 Oe. The modulating field amplitude $H_{\mathrm{ac}}$ could be varied between 0 and $20 \mathrm{Oe}$. The signals were recorded at $77 \mathrm{~K}$ for various values of the modulating field. $H_{0}$ and $H_{\mathrm{ac}}$ were collinear and perpendicular to the plane of the film. 
Table 1. Transition temperatures $T_{\mathrm{c}}$, transition widths $\Delta T$, ratios of the resistances at $300 \mathrm{~K}$ and $100 \mathrm{~K}$, and the critical current densities $J_{c}$ determined by rf absorption and transport current method for four 123 films at $77 \mathrm{~K}$.

\begin{tabular}{|c|c|c|c|c|c|c|}
\hline \multirow[b]{2}{*}{ Film } & \multirow[b]{2}{*}{$\begin{array}{c}T_{\mathrm{c}} \\
(\mathrm{K})\end{array}$} & \multirow[b]{2}{*}{$\begin{array}{l}\Delta T \\
(\mathrm{~K})\end{array}$} & \multirow[b]{2}{*}{$R_{300 /} / R_{100}$} & \multicolumn{2}{|c|}{ rf absorption } & \multirow{2}{*}{$\begin{array}{c}\text { Transport } \\
T_{\mathrm{c}} \\
\left(\mathrm{A} / \mathrm{cm}^{2}\right)\end{array}$} \\
\hline & & & & $J_{\text {eff }}$ & $J_{\mathrm{c}}$ & \\
\hline A & 86 & 2 & 2.839 & $0.15 \times 10^{2}$ & $1.86 \times 10^{5}$ & $2.3 \times 10^{5}$ \\
\hline B & 81 & $3 \cdot 6$ & $2 \cdot 846$ & $0.33 \times 10^{2}$ & $3.92 \times 10^{5}$ & $5.8 \times 10^{5}$ \\
\hline $\mathrm{C}$ & 84 & 4 & $2 \cdot 55$ & $0.34 \times 10^{2}$ & $4.02 \times 10^{5}$ & $5.0 \times 10^{5}$ \\
\hline $\mathrm{D}$ & 81 & 8 & 1.879 & $>0.1 \times 10^{3}$ & $>1 \times 10^{6}$ & not available \\
\hline
\end{tabular}

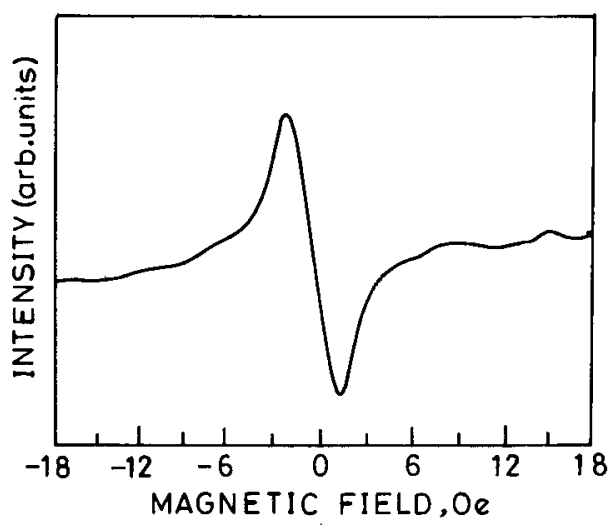

Figure 3. The derivative of the of loss signal recorded at $77 \mathrm{~K}$ for film $\mathrm{B}$.

Now we present our results on four different thin films, designated A, B, C and D for convenience, even though a much larger number of films was fabricated and studied. Table 1 presents for the four films the superconducting transition temperature $T_{\mathrm{c}}$, the transition widths $\Delta T$, the ratios of the resistances at $300 \mathrm{~K}$ and $100 \mathrm{~K}$, $R_{300} / R_{100}$, and the critical current densities at $77 \mathrm{~K}$ determined by the transport current measurement and the non-resonant $\mathrm{rf}$ absorption method ( to be discussed below). Figure 3 shows a typical non-resonant $\mathrm{rf}$ loss signal recorded in the derivative form. The peak-to-peak intensity of the signal was measured as a function of the amplitude $H_{\mathrm{ac}}$ of the modulating field. The results are plotted in figure 4 . The effects of the modulation amplitude on non-resonant microwave absorption have been studied by several workers (Bhat et al 1987b; Blazey and Hohler 1989; Pozek et al 1989; Rakvin et al 1989). Pozek et al (1989) showed that one of the effects of the modulation is to change the fluxon density in the sample. In this sense the modulating field can be considered to be superposed on the static field $H_{0}$, thus rendering the flux density inside the sample time-dependent. Following Bean (1964), the flux density inside the sample can then be written as

$$
B=B\left(H_{0}\right)+\sum_{n=1}^{\infty} \alpha_{n} \sin (n \omega t)
$$

with $\alpha_{1}$ (the amplitude of the first harmonic) $\propto H_{\mathrm{ac}}^{2} / H^{*}$ for $H_{\mathrm{ac}} \leqslant H^{*}$, where $H_{\mathrm{ac}}$ is 


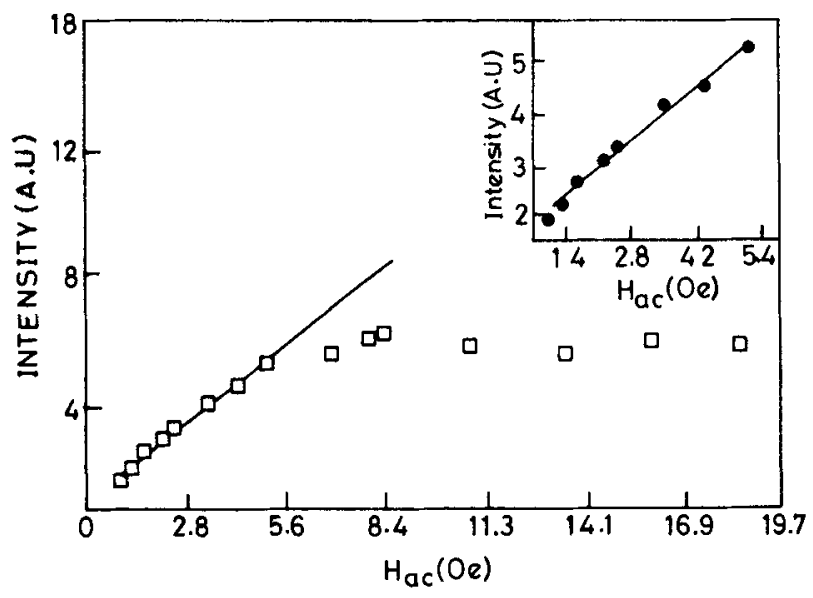

Figure 4. Intensity of the derivative signal as a function of the modulation amplitude for the film marked $B$ in table 1 . The inset shows the region of the linear fit expanded.

the amplitude of the ac field and $H^{*}$ the field value for which the flux penetrates the entire sample. The origin of the $\mathrm{r}$ loss in the case of single crystals and crystalline thin films is essentially in the damped motion of the fluxons (Srinivasu et al 1994a).

Therefore the loss

$$
S \propto f n,
$$

where a fraction $f$ of the fluxon density $n=B / \phi_{0}$, with $\phi_{0}$ the elemental flux quantum, is considered to be free. Therefore

$$
S=f\left[B\left(H_{0}\right)+\frac{H_{\mathrm{ac}}^{2}}{H^{*}} \sin (\omega t)+\text { higher harmonics }\right] .
$$

In this expression, the first term represents the dependence of the general lineshape, hysteresis and flux trapping with respect to the dc field. The second term contains the information on the modulation effects.

The maximum loss $S_{\max }$ in a modulation cycle, therefore, will be proportional to $\left(H_{\mathrm{ac}}+H_{0}\right)^{2} / H^{*}$. Since, in our experiment, consequent to lock-in detection, the derivative of the loss is recorded and therefore,

$$
\frac{\mathrm{d} S}{\mathrm{~d} H} \propto \frac{2\left(H_{\mathrm{ac}}+H_{0}\right)}{H^{*}} .
$$

Therefore, if the modulation amplitude $H_{\mathrm{ac}}$ is increased starting from a small value, until $H^{*}$ we expect a linear dependence of the recorded signal intensity on $H_{\text {ac }}$. For $H_{\mathrm{ac}} \geqslant H^{*}$, as shown by Bean, there will be a departure from the quadratic dependence of the first harmonic on $H_{\mathrm{ac}}$ resulting in the departure of the signal intensity from linearity. This is what is observed in our experiments as shown in figure 4.

Obviously, the field value for which the departure occurs corresponds to $H^{*}$. Following Bean again,

$$
H^{*}=\frac{\pi J_{c} D}{5},
$$


where $D$ is the thin film width. $J_{\mathrm{c}}$ can be calculated using this relation. However, this $J_{\mathrm{c}}$ is only an effective $J_{\mathrm{c}}$ and not the true $J_{\mathrm{c}}$ for the following reason.

As shown by Baczewski et al (1991), in calculating $J_{c}$ from the relation

$$
\nabla \times H=\frac{4 \pi J_{\mathrm{c}}}{c}=\frac{\partial H_{\mathrm{D}}}{\partial z} \frac{\partial H_{z}}{\partial D},
$$

for thin-film geometry, the term $\partial H_{\mathrm{D}} / \partial z$, i.e. the gradient of the radial field along the axial direction $z$ is the dominant one rather than the term $\partial H_{z} / \partial D$ used for calculating the $J_{\text {eff }}$ above. The term $\partial H_{\mathrm{D}} / \partial z$ is shown to exceed the $\partial H_{z} / \partial D$ by a ratio of order $D / t$ where $t$ is the thickness of the sample. Therefore the true $J_{\mathrm{c}}$ is obtained by multiplying the $J_{\text {eff }}$ by this factor. Table 1 lists values of $J_{\text {eff }}$ and $J_{\mathrm{c}}$ and also $J_{\mathrm{c}} \mathrm{s}$ determined by the transport current method. As can be seen from the table, the consistency between the $J_{\mathrm{c}} \mathrm{s}$ determined by the non-resonant $\mathrm{rf}$ absorption technique and the transport current method is quite satisfactory.

\section{Distinction between weak link and bulk responses}

One of the characteristic features of the high- $T_{\mathrm{c}}$ superconductors is the hierarchy of weak links they contain. These weak links are comprised mainly of the grain boundaries in dense powders and compact sintered pellets, whereas in isolated grains and wellannealed single crystals they originate in the twin boundaries (when present, like in the 123 crystals). In fact it is possible that due to the very short coherence length characterizing these materials, point defects, oxygen vacancies and even the insulating layers in between the conducting planes can act, in certain respects, like weak links, as originally suggested by Deutscher and Muller (1987). Then, since the lower critical field $H_{\mathrm{c} 1}$ of the weak links is orders of magnitude smaller $(\sim 0.1 \mathrm{Oe})$ than that of the bulk, the magnetic field penetration is more facile into the junctions than into the bulk material. The critical current through the junctions then becomes dependent on the field according to the well-known diffraction relation $J_{c}=J_{c}(0)\left[\sin \left(H / H_{0}\right) /\right.$ $\left.\left(H / H_{0}\right)\right]$, where the symbols have their conventional meanings, and thus can lead to the observed field-dependent absorption (Dulcic et al 1989a). In fact, this model has been proposed and successfully adapted to explain the low-field absorption behaviour by many workers (Dulcic et al 1989b; Owens 1990; Puri et al 1990). However, it is also possible when the size of the junction $d$ is such that $d>\hat{\lambda}_{\mathrm{L}}$ (where $\hat{\lambda}_{\mathrm{L}}$ is the London penetration depth) fluxons are nucleated within the weak links and their viscous motion will result in dissipation. This model was proposed by Portis et al (1988) to explain the low-field absorption in pellet samples.

It has been realized that to distinguish between these two different possible origins of the non-resonant microwave absorption signals is not easy (Dulcic et al 1989c). Further, the contribution to the dissipation from the Abrikosov fluxons and from the fluxons nucleated in the intragranular weak links (twin boundaries, insulating layers in the structure, and the like), if any, also need to be delineated. Here we show that studying the non-resonant absorption at widely different frequencies can help decide between the diffraction effect and the viscous fluxon motion as the possible causes of dissipation. We have studied the YBCO superconductor in two different forms, namely epitaxially grown thin films and sintered pellets, in both the microwave $(9 \cdot 1 \mathrm{GHz})$ and radio $(19 \cdot 5 \mathrm{MHz})$ frequency ranges. We observe characteristic depen- 
dence of the lineshapes on frequency and the nature of the samples, from which we conclude that close to $T_{\mathrm{c}}$ in the thin-film and the sintered-pellet forms the lineshapes are more consistent with the model of dissipative fluxon motion and not that of the diffraction effect. While there have appeared a large number of reports on the study of non-resonant absorption using EPR spectrometers there are hardly any such measurements in the rf range [apart from the early report of Bhat et al (1987b), the only other work appears to be Orlandi and Rigamonti (1991)]. We show (Srinivasu et al 1994a), for the first time, how a comparative study covering the two frequency ranges can give insight into the microscopic origin of the dissipative processes. For a taste of the rich panorama of the properties of the high- $T_{\mathrm{c}}$ superconductors as revealed by the method of non-resonant electromagnetic response the reader is referred to some of our other publications (Bhat et al 1991, 1994; Srinivasu et al 1994b).

The sintered pellets of the YBCO samples used were prepared by the conventional solid-state method. The thin films of the same were prepared by the pulsed laser deposition technique as described in Hwang et al (1989). The films were deposited on $\mathrm{SrTiO}_{3}(100)$ substrates (kept at $\sim 740^{\circ} \mathrm{C}$ ) and had the $c$ axis perpendicular to the plane of the film. Thickness of the films was measured to be $\sim 2500 \AA$. A fourprobe resistivity measurement indicated a $T_{\mathrm{c}}$ of $87 \mathrm{~K}$ with the transition width of $\sim 2 \mathrm{~K}$. The critical current density $J_{\mathrm{c}}$ was estimated to be $\sim 5 \times 10^{5} \mathrm{~A} / \mathrm{cm}^{2}$ at $77 \mathrm{~K}$ showing that the film was of moderately good quality. An optical polarizing microscope examination showed that similarly prepared films were free from intergranular boundaries though twin boundaries were present. It may further be noted that films prepared following the same method and with $J_{\mathrm{c}} \sim 7.5 \times 10^{5} \mathrm{~A} / \mathrm{cm}^{2}$ have earlier been shown to be free from intergranular junctions (Hwang et al 1989).

The non-resonant absorption experiments in the microwave range were carried out with a Varian E109 X-band EPR spectrometer operating at a nominal microwave frequency of $9 \cdot 1 \mathrm{GHz}$, using mostly $100 \mathrm{kHz}$ field modulation and phase-sensitive detection. It is well known (Bhat et al 1987b; Blazey and Hohler 1989; Pozek et al 1989; Rakvin et al 1989) that in the low-modulation regime $(\sim 0.05 \mathrm{Oe})$ the signals are dominated by the modulation of the boundary currents, giving rise to opposite phases of the signals for forward and reverse magnetic field sweeps. However, at higher modulation amplitudes, the magnetic flux density gets modulated, resulting in signals which are true derivatives of the absorption. Therefore the modulation amplitude in our experiments was fixed at 4 Oe for all the experiments ensuring that the modulation amplitude-dependent hysteresis and phase reversal do not complicate the results. For the modulation amplitudes used in these experiments, we are in the regime where the signal intensity is proportional to the modulation amplitude. It was checked and confirmed that the signal shapes are not distorted at this and even a slightly higher $(5 \mathrm{Oe})$ value of the modulation amplitude. A microwave power of $\sim 22 \mathrm{~mW}$ is used throughout. For the experiments in the rf range, a home-made (CW) NMR spectrometer (Bhat et al 1987b) operating at a nominal frequency of $19.5 \mathrm{MHz}$ was used. Fixed modulation of $4 \mathrm{Oe}$ at a frequency of $87 \mathrm{~Hz}$ and phasesensitive detection were used.

In both the microwave and rf ranges the experiments were carried out at $77 \mathrm{~K}$, by directly immersing the samples in liquid nitrogen. All the recordings were done with the $a b$-plane of the film perpendicular to the static field (i.e. $H \| c$ ). The magnetic field was mostly scanned between $-50 \mathrm{Oe}$ and +50 Oe thus eliminating any scan rangedependent phase reversal and hysteresis or other artifacts (however, see below). 


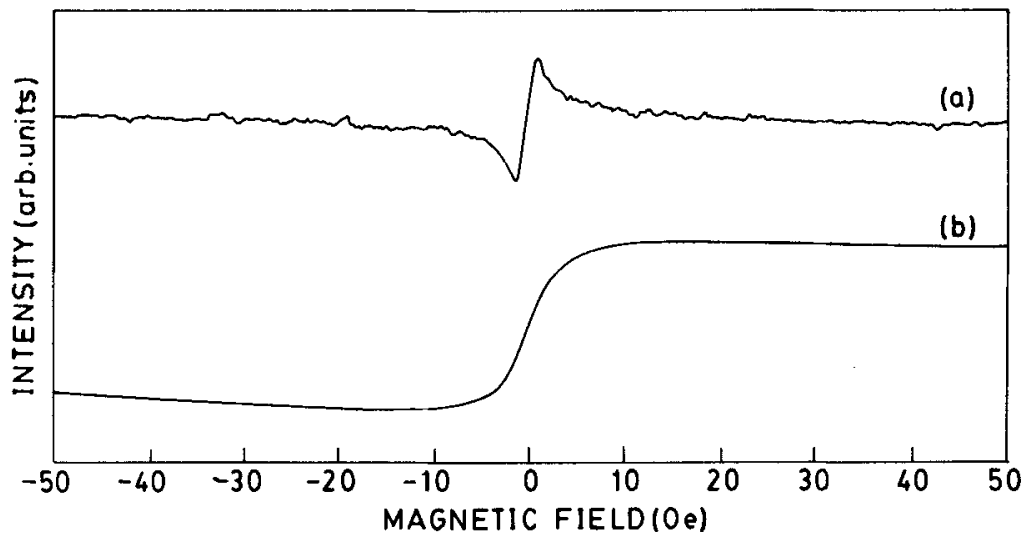

Figure 5. Field derivative of the non-resonant absorption signals for the YBCO thin film at (a) $19.5 \mathrm{MHz}$ and (b) $9.1 \mathrm{GHz}$. To be noted is the drastic difference in the lineshapes at the two frequencies.

With these experimental conditions, the forward and backward sweeps of the static magnetic field produced almost reversible derivative absorption signals in both the microwave and $\mathrm{rf}$ ranges for $H \perp a b$-plane of the film. Figure 5 presents the field derivatives of the non-resonant absorption signals recorded at $19.5 \mathrm{MHz}$ and $9 \cdot 1 \mathrm{GHz}$ for the thin-film samples. The line shapes are seen to be quite different in the two cases. Derivative-like signals reaching the baseline at a static field $\sim 20$ Oe indicating a saturation of the field-dependent absorption at low static fields are observed in the rf range, whereas in the microwave range the signals do not come back to the baseline until at much higher fields, proceeding almost in a parallel fashion to the field axis at low fields. Usually, the magnetic field is modulated at a frequency of $100 \mathrm{kHz}$ in commercial EPR spectrometers while we had used $87 \mathrm{~Hz}$ for the experiments in the rf range. To eliminate the possibility of the observed difference in the lineshapes arising due to the difference in the modulation frequency, experiments were carried out using $87 \mathrm{~Hz}$ as the modulating frequency with the EPR spectrometer. No difference in the lineshape from that obtained with $100 \mathrm{kHz}$ modulation was observed establishing that the difference in the lineshapes is not due to the difference in the modulation frequencies. In figure 6 we show signals from thin-film and pellet samples recorded at $9 \cdot 1 \mathrm{GHz}$. It is seen that while in the case of the thin film the signal does not saturate until very high fields (1-2 kOe) (results not shown), in pellet samples they saturate at much lower $(20 \mathrm{Oe})$ fields. Such absorption signals in the microwave range extending to large fields, similar to our observation in thin films, were earlier observed in YBCO single crystals in a small temperature range just below $T_{\mathrm{c}}$ by Dulcic et al (1988) (see the ' $A$ ' type signals in their figure 1). Further, these signals also were shown to have no hysteresis and therefore were true derivatives of absorption. Similar signals were reported by Buluggiu et al (1991) in compact sintered pellets of YBCO and bismuth 2212 samples and by Kataev et al (1991) in bismuth 2212 samples. The results of Marcon et al (1991) on the field dependence of non-modulated microwave absorption in YBCO sintered pellets are also similar. An important conclusion coming from these various studies is that the microwave absorption goes on increasing with the magnetic field up to high fields in sintered compact pellets and isolated powders, but 


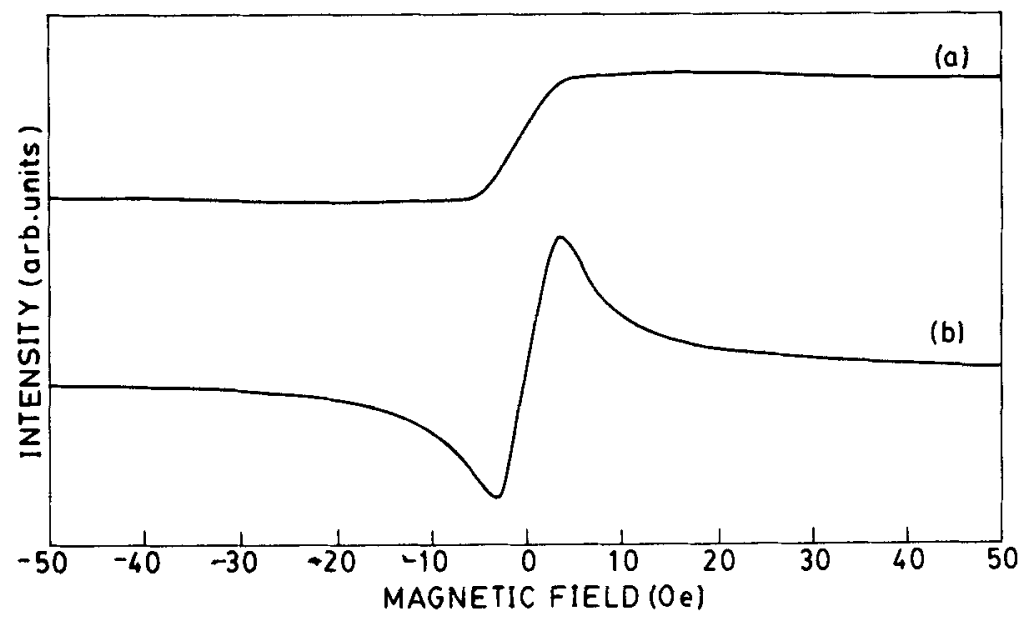

Figure 6. Field derivative of the non-resonant microwave absorption signal $(\omega=9 \cdot 1 \mathrm{GHz})$ for (a) the YBCO thin film and (b) the YBCO pellet.

saturates at very low fields of the order of $10-50$ Oe in loosely packed pellets and powders that are not crushed to submicron size and are not isolated from each other.

Dulcic et al (1989a) attribute their type A signals in single crystals to the microwave loss arising due to the diffraction reduction of junction currents $J_{c}$ in twin boundaries because of the applied magnetic field. According to this model the power absorbed in the junction, $P$, takes the form

$$
P=P_{\mathrm{n}} \frac{1}{1+\eta_{\mathrm{D}}},
$$

where $P_{\mathrm{n}}=(1 / 2) I_{\mathrm{mw}}^{2} R$ is the absorption when the junction is driven normal. $I_{\mathrm{mw}}$ is the microwave current, $R$ the junction resistance, and

$$
\eta_{\mathrm{D}}=\eta_{0} F^{2}(H)
$$

where $\eta_{0}$ is the value of the parameter $\eta$ at zero magnetic field. $F(H)$ is the value of the reduction factor $\left[\sin \left(H / H_{0}\right)\right] /\left(H / H_{0}\right)$ averaged over all junctions and $H_{0} \approx$ $\left(\phi_{0} / \mu d L\right)$, wherè $\mu$ is the permeability, and $d=2 \lambda_{L}+t$ for a junction of thickness $t$ and length $L$. Thus in this model, the junction supercurrent decreases as the applied magnetic field increases leading to an increase in the microwave absorption. The field $H_{0}$ at which the absorption saturates is seen to depend upon the junction dimensions. Following the above model, Dulcic et al (1989a) explain the large saturation fields ( 1-3 kOe) observed for their type A signals as originating in the junctions formed by the twin boundaries of dimensions $10^{3}-10^{4} \AA$. Our experimental results show that for the thin-film sample of YBCO, microwave absorption at $9 \cdot 1 \mathrm{GHz}$ does not saturate until the applied fields reach values as high as $\sim 1 \mathrm{kOe}$, whereas at $19.5 \mathrm{MHz}$ the absorption saturates at very low fields $(\sim 20 \mathrm{Oe})$ as shown in figure 5 , with all the other experimental conditions remaining the same. Therefore the model used by Dulcic et al (1988), where the saturation field depends upon the weak-link dimensions, cannot explain this result where just by changing the frequency from $9 \cdot 1 \mathrm{GHz}$ to $19.5 \mathrm{MHz}$ 
we see saturation fields differ by orders of magnitude. The expected frequency dependence of absorption from the Dulcic model (figure $2 b$ of Dulcic et al 1989a) is in fact opposite to our observations, i.e. according to the model of the microwave absorption originating in the diffraction effect, the width of the absorption decreases with increasing frequency, whereas we find experimentally that in the $\mathrm{rf}(19.5 \mathrm{MHz})$ range, the derivative of the absorption signal is narrow and by increasing the frequency to microwave range $(9 \cdot 1 \mathrm{GHz}$ ), the signal width increases (figure 5). Therefore we believe that in the small temperature range below $T_{c}$, the field dependence of the microwave absorption in single crystals and single-crystalline YBCO films does not arise because of the diffraction effect, but, as will be shown below, because of the damped motion of free fluxons. We show that the large frequency dependence of the lineshape observed experimentally follows from this model, which was earlier used by Portis et al (1988).

Firstly, the near absence of hysteresis both in the microwave and the radio frequencies suggests that we are almost completely in the flux flow regime. It may be mentioned that earlier Marcon et al (1991) attributed this kind of hysteresis-less signals near $T_{\mathrm{c}}$ to flux flow. In such a situation we are justified in taking $B \sim H$. Then, the surface resistance $R$ arising from the damped motion of the fluxons is given by

$$
R=\left(X_{0} / \sqrt{2}\right)\left(-1+\left(1+4\left(f / B_{0}\right)^{2} B^{2}\right)^{1 / 2}\right)^{1 / 2},
$$

where $X_{0}=\left[4 \pi \omega \mu \lambda_{L} / c^{2}\right] \mu^{1 / 2}$ is the impedance at zero magnetic field, $B$ the flux density, and $f$ the fraction of free fluxons. The permeability $\mu$, defined by the relation $B=\mu H$, is $\sim 1$ and $\omega$ is the frequency of the electromagnetic radiation. The fraction of free fluxons $f$ was taken by Portis et al to be $\sim 0 \cdot 1$. However, in the flux flow regime, as obtained in our experiment, one can reasonably assume that all the fluxons are free and therefore $f \sim 1$. Further, in (9) $B_{0}$ is given by

$$
B_{0}=8 \pi \omega \mu \eta \lambda_{L}^{2} / \phi_{0},
$$

where $\eta$ is the coefficient of viscosity, $\phi_{0}$ the elementary flux quantum, $\lambda_{L}$ the London penetration depth, and $\mu$ the permeability taken to be $\sim 1$ as explained above. With this, $B_{0}$ can be written as

$$
B_{0}=8 \pi \omega \eta \lambda_{L}^{2} / \phi_{0},
$$

and thus essentially depends on the frequency, the viscosity coefficient and the penetration depth. Since at $77 \mathrm{~K}$ both if and microwaves penetrate the thin film (of thickness $\sim 2500 \AA$ ) fully, $\lambda_{L}$, which is the same for both the frequency ranges (estimated (Krusin-Elbaum et al 1989) to be $\sim 2000 \AA$ at $77 \mathrm{~K}$ ) does not alter the conclusions regarding the frequency dependence of the lineshapes of the signals from a given film. Therefore a change in either $\omega$ or $\eta$ changes $B_{0}$. By plotting (9) for different values of $f / B_{0}$, (in our case $f \sim 1$ ), i.e. essentially for different values of $B_{0}$, we can see from figure 7 that the derivative of absorption vs field shows different characteristics observed experimentally. In this figure with the dc field swept between $-50 \mathrm{Oe}$ and $+50 \mathrm{Oe}$, the field derivative of the absorption is plotted for different $B_{0}$ values ranging from $10 \mathrm{Oe}$ to $1000 \mathrm{Oe}$. One can observe clearly that for small values of $B_{0}$ of the order of $\sim 10 \mathrm{Oe}$, the absorption signals saturate quickly, whereas for $B_{0}=1000 \mathrm{Oe}$, the signal does not saturate at low fields. Experimentally, for a 


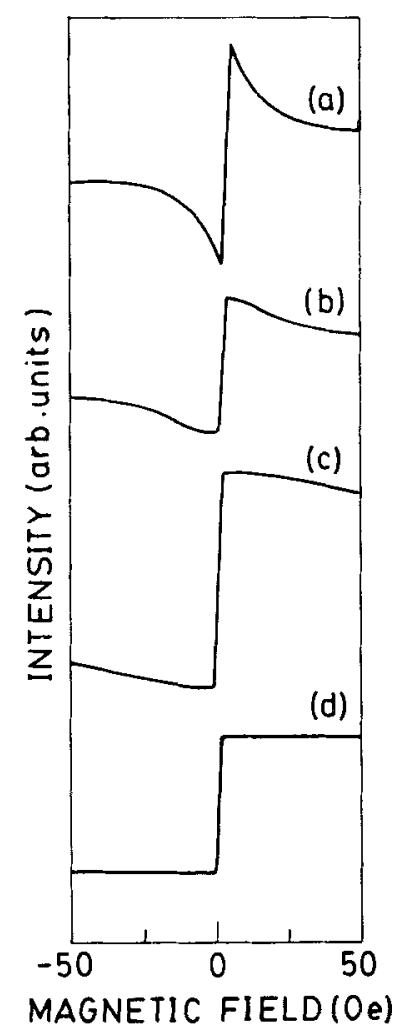

Figure 7. Simulation of the field derivative of the non-resonant absorption signals for different values of $B_{0}:$ (a) $10 \mathrm{Oe}$, (b) $30.3 \mathrm{Oe}$, (c) $100 \mathrm{Oe}$ and (d) $1000 \mathrm{Oe}$.

given sample with a certain $\eta$ different values of $B_{0}$ are realized by changing the operating frequency. As mentioned earlier, in our experiment we have recorded the signals at $9.1 \mathrm{GHz}$ and $19.5 \mathrm{MHz}$. The corresponding spectra shown in figure 5 in the derivative form were taken at the same temperature and with field sweeps of $\pm 50 \mathrm{Oe}$. One can see that at $19.5 \mathrm{MHz}$ the signal saturates at $\sim 20 \mathrm{Oe}$, whereas at $9.1 \mathrm{GHz}$ it does not saturate at low fields. These experimentally observed derivative absorption lineshapes at the two widely different frequencies can be simulated using (9), with appropriate $B_{0}$ values, e.g. $B_{0} \sim 1 \mathrm{Oe}$ for $\mathrm{rf}$ and $150 \mathrm{Oe}$ for microwave frequency. Figure 7 shows such simulation for the rf range. For this simulation we have used $\omega=19.5 \mathrm{MHz}, \lambda_{L} \sim 2000 \AA$ (Krusin-Elbaum et al 1989) and $B_{0}=1$ gauss which gives $\eta \sim 0.878 \times 10^{-6} \mathrm{cgs}$ units. This value of $\eta$ is quite high and rules out any grain-boundary effects following Portis and Blazey (1985), who argue that grainboundary viscosity coefficient is of the order of $10^{-13} \mathrm{cgs}$ units. The high viscosity coefficient obtained by us which is comparable with the bulk values suggests that the low-field microwave absorption in our thin-film samples is coming from the bulk. Recently Kataev et al (1991) showed that in very compact pellets signals saturate at high fields as in the case of single crystals and thin films. One can understand this result in the following way: in a compact pellet, flux squeezing effects (Dulcic et al 1989c; Orlandi and Rigamonti 1991) dominate and even low applied fields can enter into grains due to these effects. Once flux enters into the grains, one would see 
intragranular effects which would again make the signals look similar to those in films and single crystals.

Another possibility that needs to be considered is that of the intrinsic weak links (Deutscher and Muller 1987; Dulcic et al 1988, 1989 ) present even in single crystals and single-crystalline thin films. Some of these weak links can conceivably have superior qualities like high values of $\eta$ showing microwave absorption signals similar to that of the bulk. But recent magnetization measurements on thin-film (Yeshurun et al 1990) as well as single-crystal (Tamegai et al 1992) sumples show the applicability of Bean's critical-state model for these cases ruling out granularity even on the scale of the twin boundaries. Another important point about the losses due to damped motion of free fluxons in weak link is that the weak link size should be larger than the fluxon size, so that the fluxon can move inside the weak link. Certain defects like point defects and oxygen vacancies cannot contribute to this mode of loss because the fluxons cannot move inside such defects and therefore such defects can only act as pinning centres. On the basis of all these considerations it seems very likely that the microwave absorption in our thin-film samples which saturates at high magnetic fields has its origin in the motion of the fluxons in the bulk of the material.

\section{Acknowledgements}

The work reported here is the result of fruitful collaboration with Prof. C N R Rao, Prof. N Kumar, Prof. T V Ramakrishnan, Prof. P Ganguly, Prof. M S Hegde, Dr N Y Vasanthacharya, Dr Boben Thomas, Mr V V Srinivasu and Mr Amit Rastogi. The Department of Science and Technology is thanked for providing financial support.

\section{References}

Abragam A 1961 The principles of nuclear magnetism (Oxford: Clarendon Press)

Andrew E R 1955 Nuclear magnetic resonance Camibtidge Monographs in Phystics (Cambridge: Cambridge University Press)

Baberschke K 1976 Z. Physik. B24 53

Baczewski L T, Piotrowski K, Szymczak R, Szymeżdk H ảnd Malozemoff A P 1991 Physica C175 363

Bean C P 1962 Phys. Rev. Lett. 8250

Bean C P 1964 Rev. Mod. Phys: 3031

Bednorz J G and Mullef K A 1986 Z. Phys. B64 189

Bhat S V. Ganguly P and Rao C N R 1987a Pramana-J. Phys. 28 L425

Bhat S V, Ganguly P, Ramakrishnan T V and Reo C N R 1987b J, Phys. C. Solid State Phys. 20 L559

Bhat S V, Rastogi A, Kumar N, Nagarajät R and Rao C N R 1994 Phyulca C219 87

Bhat S V, Srinivasu V V and Kumar N 1991 thys. Rev. B44 10121

Blazey K A 1990 in Springet series in solid state seience: Earflef and tecent aspects of superconductivity (eds) J G Bednorz and K A Mullè (Berlin: Spfínget) vol. 90 , p. 262

Blazey K W and Hohler A 1989 Solid State Commun. 721199

Blazey K W, Muller K A, Bednorz J G, Berlinger W, Amoretti G, Buluggiu E, Vera A and Matacotta F C 1987 Phys. Rev. B36 7241

Blazey K W, Portis A M and Bednorz J G 1988 Solid State Commun. 651153

Buluggiu E, Vera A, Giori D C, Amoretti G and Licci F 1991 Supercond. Sci. Technol. 4595

Chu C W et al 1987 Phys. Rev. Lett. 58405

Deutscher G and Muller K A 1987 Phys. Rev. Lett. 591745

Dulcic A, Crepeau R H and Freed J H 1988 Phys. Rev. B38 5002

Dulcic A, Rakvin B and Pozek M 1989a Europhys. Lett. 10593 
Dulcic A, Crepeau R H and Freed J H 1989b Physica C160 213

Dulcic A, Crepeau R H and Freed J H 1989c Phys. Rev. B39 4249

Dulcic A, Leontic B, Peric M and Rakvin B 1987 Europhys. Lett. 41403

Durney R, Hautala J, Ducharme S, Lee B, Symco O G, Taylor P C, Zeng D J and Xu J A 1987 Phys. Rev. B36 2361

Hwang D M, Venkatesan T, Chang C C, Nazar L, Wu K D, Inam A and Hegde M S 1989 Appl. Phys. Lett. 541702

Kataev V, Knauf N, Buchner B and Wohllebeb D 1991 Physica C184 165

Khachaturyan K, Weber E R, Tejedor P, Stacy A M and Portis A M 1987 Phys. Rev. B36 8309

Krusin-Elbaum L, Greene R L, Holtzberg F, Malozemoff A P and Yeshurun Y 1989 Phys. Rev. Lett. 62 217

Marcon R, Fastampa R, Guira M and Silva E 1991 Phys. Rev. B43 2940

Mehran F and Anderson P W 1989 Solid State Commun. 7129

Orlandi P and Rigamonti A 1991 Physica C178 197

Owens F J 1990 Physica C171 25

Peric M, Rakvin B, Prester M, Brnicevic N and Dulcic A 1988 Phys. Rev. B37 522

Pilbrow J R 1990 Transition ion EPR (Oxford: Clarendon Press)

Poole C P 1983 Electron spin resonance, A comprehensive treatise on experimental techniques (New York: Wiley Interscience)

Portis A M and Blazey K W 1988 Solid State Commun. 681097

Portis A M, Blazey K W, Muller K A and Bednorz J G 1988 Europhys. Lett. 5467

Pozek M, Dulcic A and Rakvin B 1989 Solid State Commun. 70889

Puri M, Masiakowski J T, Marrelli S, Bear J and Kevan L 1990 J. Phys. Chem. 946094

Rakvin B, Pozek M, Paljevic M and Brnicevic N 1989 Solid State Commun. 70729

Rettori C, Davidov D, Belaish I and Felner I 1987 Phys. Rev. B36 4028

Srinivasu V V, Boben Thomas, Vasanthacharya N Y, Hegde M S and Bhat S V 1991 Solid State Commun. 79713

Srinivasu V V, Boben Thomas, Hegde M S and Bhat S V 1994a J. Appl. Phys. 754131

Srinivasu V V, Bhat S V and Kumar N 1994b Solid State Commun. 89375

Stankowski J, Kahol P K, Dalal N S and Moodera J S 1987 Phys. Rev. B36 7126

Tamegai T, Krusin-Elbaum L, Civale L, Santhanam P, Brady M J, Masselink W T, Holtzberg F and Feild C 1992 Phys. Rev. B45 8201

Yeshurun Y, McElfresh M W, Malozemoff A P, Hagerhorst-Trewhella J, Mannhart J, Holtzberg F and Chandrasekhar G V 1990 Phys. Rev. B42 6322 